\title{
2-Octyl cyanoacrylate adhesive for the prevention of central venous catheter infections:A proof of concept
}

\begin{abstract}
Introduction: Colonization of central venous catheters (CVC)s by microorganisms is an event that precedes central line associated bloodstream infection (CLABSI). It is plausible that sealing the skin insertion site of CVCs with 2-octyl cyanoacrylate adhesive may reduce catheter colonization by creating a sterile barrier and minimizing subsequent extraluminal colonization of the catheter. We explored this by evaluating the ability of the skin adhesive to maintain a sealed interface by visual inspection.
\end{abstract}

Materials and methods: 19 enrolled patients underwent application of 2-octyl cyanoacrylate adhesive to the CVC insertion site, followed by daily inspection for visual signs of 2-octyl cyanoacrylate loss of seal integrity with the skin/catheter interface. Additionally, patients were monitored daily for local cutaneous reactions and/or catheter malfunction. After the clinical team removed the catheter, the tip was sent to the microbiology lab for culture via roll plate method.

Results: Seal integrity by visual inspection was maintained for a range of 24 days (1-25 days), mean of 6.9 days a median of 7.0 days, and a mode of 7 days. Among the 4 catheters that were cultured in the microbiology laboratory one demonstrated bacterial colonization.

Conclusion: 2-octyl cyanoacrylate sealed the skin/catheter insertion site for about 7 days, by visual inspection. Further investigation is necessary to determine if use of this skin adhesive leads to reductions in the rate of catheter colonization and CLABSI.

Keywords: cardiovascular anesthesiology, critical care and resuscitation, quality improvement
Volume 9 Issue 2 - 2017

\author{
Aaron B Dahl,' Zachary Cohen,' David K \\ Warren, ${ }^{2}$ Thomas J Graetz' \\ 'MD,Washington University School of Medicine Department of \\ Anesthesiology, USA \\ ${ }^{2} \mathrm{MD}, \mathrm{MPH}$, Washington University School of Medicine \\ Department of Medicine, USA
}

Correspondence: Aaron B Dahl, MD,Washington University School of Medicine, Department of Anesthesiology, 660 South Euclid Ave, Campus Box 8054, St. Louis, MO 631 I0, USA, Tel 360-739-8906, Fax 3|4-747-3977, Email abdahl@wustl.edu

Received: November 0I, 2017 | Published: November 24, 2017
Abbreviations: CLABSI, central line associated bloodstream infection; $\mathrm{CVC}$, central venous catheter; $\mathrm{CFU}$, colony forming units

\section{Introduction}

Colonization of central venous catheters (CVC)s by microorganisms is an event that precedes central line associated bloodstream infection (CLABSI). The average rate of CLABSI among critically ill patients in the United States is 0.9-3.4 infections per 1000 catheter days. ${ }^{1,2}$ With 250,000 cases of CLABSI occurring annually and costing $\$ 6000$ to $\$ 90,000$ per event, there is significant interest in reducing this occurrence. ${ }^{2,3}$ CLABSI originates from both intraluminal and extraluminal sources. Extraluminal colonization is thought to be due to organisms present at the catheter insertion site and likely reflects the quality of aseptic technique utilized upon catheter insertion in addition to insertion site care. ${ }^{4}$ Daily maintenance of the sterility of the catheter insertion site is an intervention that may be underappreciated. Currently recommended catheter insertion site maintenance techniques for the prevention of CLABSI involve using either sterile gauze or sterile, transparent, semipermeable dressings to cover the site. ${ }^{5}$

An adjuvant to standard insertion site dressings is to target the catheter-tissue interface with a tissue adhesive, specifically

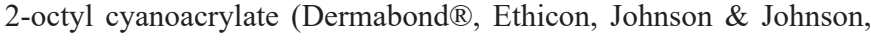
Somerville, NJ), which is applied at the time of catheter insertion. Standard securement with sutures and semipermeable dressings still allow backwards and forwards motion of the catheter in and out of the skin. This "pistoning" may promote bacterial entry into the cannulated vein. ${ }^{6,7} 2$-octyl cyanoacrylate may potentially decrease the "pistoning" effect of the catheter.

Since approval by the FDA in 1998 for the clinical use of 2-octyl cyanoacrylate as a topical skin adhesive there has been widespread adoption of this product in the care of surgical and trauma patients for wound closure. ${ }^{8}$ with subsequent proven efficacy in decreasing surgical site infections in vivo when used on surgical wounds. ${ }^{6,9-}$ ${ }^{11}$ Additionally, An in vitro study from Simonova et al. ${ }^{6}$ showed no bacterial growth under 16 GA 1.16 " $(1.7 \times 30 \mathrm{~mm})$ Insyte $^{\mathrm{TM}}$ Autoguard $^{\mathrm{TM}}$ (BD, Franklin Lakes, NJ, USA) Intravascular catheters applied with tissue adhesive. ${ }^{6}$ While 2-octyl cyanoacrylate is not FDA approved for the use of securing CVCs, the off-label use of a tissue adhesive for securement of CVCs was first reported in 2007 in 30 burn patients ${ }^{7}$ with the same group reporting successful use of this adhesive for securing 50 thoracic epidural catheters. ${ }^{11}$ We investigated the utilization of this product for the securement of CVCs off-label.

The objectives of this study were four-fold;1) Investigate the chemical and ultra-structural changes that could occur to a $7 \mathrm{Fr}$. triple lumen $20 \mathrm{~cm}$ radiopaque polyurethane with blue FlexTip ${ }^{\circledR}$ ARROWgard Blue PLUS ${ }^{\circledR}$ catheters with antimicrobial surface treatment (Reading, PA, USA) with 2-octyl cyanoacrylate tissue adhesive use. 2) Determine the ability of 2-octyl cyanoacrylate tissue adhesive to adhere to the skin catheter interface. 3) Identify the duration that 2-octyl cyanoacrylate will adhere to the skin and catheter and maintain a mechanical barrier. 4) Identify catheter tip bacterial colonization with 2-octyl cyanoacrylate tissue adhesive use. Catheter colonization was defined as growth of 15 or more CFU 
of bacteria using the roll plate method.$^{12}$. We hypothesized that a 2-octyl cyanoacrylate sealed catheter/skin interface would decrease colonization by bacteria.

\section{Materials and methods}

This study was conducted at Barnes-Jewish Hospital, an academic hospital in affiliation with Washington University Medical Center located in the United States following Institutional Review Board approval. Written informed consent was obtained from all subjects prior to enrollment. The study design, data collection, and data presentation was without influence or consultation from the manufacturer of the tissue adhesive. Neither industry monetary funding nor product support was provided for this study. Patients received all study interventions without charge. Prior to patient enrollment in collaboration with the Washington University Department of Mechanical Engineering and Materials Science we utilized Raman spectroscopy and Electron microscopy to assess for any potentially negative ultrastructural and/or chemical interactions between the catheter and the tissue adhesive. This was accomplished by exposing the catheters to the 2-octyl cyanoacrylate for a period of 10 days and then performing the material analysis prior to and following removal of the adhesive with acetone.

Recruitment occurred between September 2015 and June of 2017 by screening the daily surgical schedule to identify patients undergoing procedures potentially receiving a multilumen central venous catheter. If a multilumen catheter was part of the attending anesthesiologist's pre-existing anesthetic plan for central venous access, then the patient was eligible for inclusion and was subsequently evaluated for exclusion criteria and approached for consent. Subjects 18 years of age and older with plans to receive a multilumen subclavian or internal jugular central venous catheters were recruited from the preoperative holding areas or as inpatients awaiting surgery.

Exclusion criteria included; pregnant women, patients with catheters placed under emergent conditions, patients likely not to survive 3 days, those with skin disorder at the site of insertion, allergy to tissue adhesives or petroleum jelly, and patients unable to provide informed consent. Known risks of the adhesive such as skin reactions remain generally minor and were explained to the patient. These rare side effects include reports of contact dermatitis and localized foreign body reactions. ${ }^{13-15}$ All catheters were placed in the operating room using standard sterile technique in the internal jugular or subclavian vein. After routine insertion of the multilumen central venous catheter, the proceduralist was supplied with a sterile vial of 2-octyl cyanoacrylate and, under direct supervision of the research team, applied the adhesive to the base of the central venous catheter at the skin/catheter interface. Once the 2-octyl cyanoacrylate was applied, the proceduralist was instructed to suture the catheter in standard fashion and a transparent semipermeable dressing was applied (Figure 1).

The research team performed daily visual inspection of the multilumen catheters and documented the findings. When dressing changes occurred every 72 hours as per institutional policy, the nursing staff was instructed to call a member of the research team to inspect and document the integrity of the 2-octyl cyanoacrylate seal in the absence of the transparent dressing. Once the visual seal integrity was lost the patient was observed but no further interventions were performed, and there was no further daily documentation or photographs completed. The primary outcome measured was the day at which the 2-octyl cyanoacrylate fractured (i.e., no longer visibly adherent to both the skin and/or the catheter). The secondary outcomes measured were catheter colonization rates by roll plate method, site reactions (localized eruption, blistering, erythema, purulence or other evidence of localized untoward effects from the 2-octyl cyanoacrylate or the standard semipermeable dressing) and any CVC malfunction leading to catheter removal or replacement.

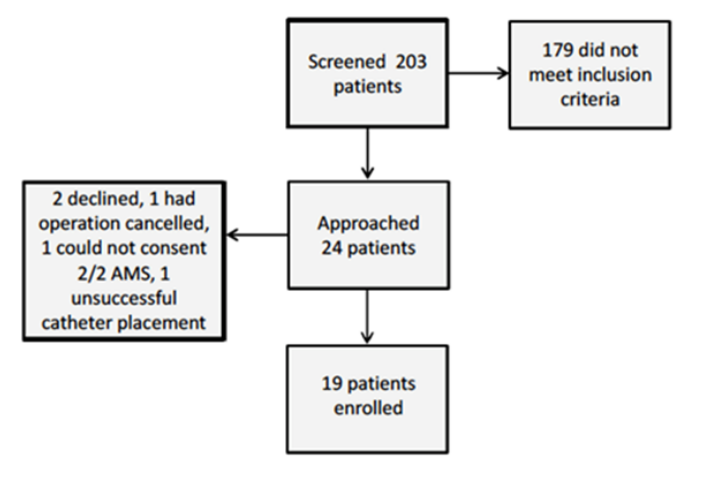

Figure I Flowchart of the screening, exclusion and enrollment process for our study.

The following additional information was obtained for all subjects: site of catheter insertion, dates of catheter placement and removal, occurrence of difficulties and violations of aseptic technique during insertion or removal, and the reason for catheter removal. The clinical daily photographs taken were made available for evaluation by a dermatologist to determine the type of reaction present, if any were suspected. As this was not a blinded intervention there was no methodology to avoid interpretation bias other than the communication of a standardized monitoring and catheter removal protocol to the nursing staff.

\section{Results}

Regarding the material analysis that took place prior to patient enrollment; Raman spectroscopy and Electron microscopy demonstrated no chemical or structural changes to the catheter from the 2-octyl cyanoacrylate adhesive following its removal with acetone after a 10-day dwell period (Figure $2 \& 3$ ). Following our material analysis, two hundred and three patients were screened; of these, 24 patients were planned to have a CVC placed in the OR and were approached for consent. Of the patients approached, 2 declined enrollment, 1 patient's operation was cancelled for nonmedical reasons, 1 patient had an attempted but unsuccessful CVC placement, and 1 patient did not possess decision making capacity to consent for enrollment. 19 patients were successfully enrolled in our study (Figure 4). Seal integrity was maintained for a range of 24 (1-25 days), mean of 6.94 days a median of 7 days, and a mode of 7 days when excluding one patient with an unidentified loss of seal integrity date (Table 1 $\& 2$ ). Attempts were made to send all catheters to the microbiology lab for culture following removal. Among the catheters delivered to the microbiology lab (some were not delivered due to a variety of circumstances, e.g., unintended removal by patient/clinical staff and intra-op death) four catheters were successfully processed and one grew S. epidermidis. The catheter that grew S. epidermidis removed lost seal integrity within the first day of catheter placement and was removed on post-operative day 1 .

Five patients (Patients 1, 2, 7, 12, and 17) enrolled in the study ultimately died. The causes of death were related to intraoperative cardiac arrest and multisystem organ failure, none were attributable to the central line or the 2-octyl cyanoacrylate. Selection bias was 
inherent in the design of the study as multilumen catheters were often placed in high risk patients with multiple pre-existing comorbidities who were undergoing complex cardiothoracic operations with anticipated prolonged ICU courses.

Table I Patient data I

\begin{tabular}{|c|c|c|c|c|c|c|c|}
\hline Patient & Age & Sex & Diagnosis & Operation & Seal duration & Culture results & Complications \\
\hline 1 & 96 & $\mathrm{~F}$ & AS & TAVR & I & $\mathrm{N} / \mathrm{A}$ & Intraop Death \\
\hline 2 & 86 & $\mathrm{~F}$ & AS & TAVR & 7 & No Growth & $\begin{array}{l}\text { Post-op death on ECMO } \\
\text { for Cardiogenic Shock }\end{array}$ \\
\hline 3 & 61 & M & $\begin{array}{l}\text { Prosthetic MV } \\
\text { Thrombosis }\end{array}$ & MVR; ECMO; IABP & 3 & N/A & \\
\hline 4 & 65 & M & $\mathrm{CHF}$ & LVAD & 7 & $\mathrm{~N} / \mathrm{A}$ & \\
\hline 5 & 58 & M & $M R$ & MAZE; MV Repair & 8 & $\mathrm{~N} / \mathrm{A}$ & \\
\hline 6 & 68 & $\mathrm{~F}$ & $\mathrm{CHF}$ & LVAD & 8 & $\mathrm{~N} / \mathrm{A}$ & \\
\hline 7 & 63 & $\mathrm{~F}$ & TAA & TAA Repair & 4 & $\mathrm{~N} / \mathrm{A}$ & Patient Death \\
\hline 8 & 52 & $\mathrm{~F}$ & CHF;TR; MR & $\begin{array}{l}\text { MV/TV Ring;ASD closure; LAA } \\
\text { ligation; MAZE }\end{array}$ & 4 & N/A & \\
\hline 9 & 74 & $\mathrm{~F}$ & $\begin{array}{l}\text { Ascending Aortic } \\
\text { Arch Aneurysm }\end{array}$ & $\begin{array}{l}\text { Ascending \& Transverse Aortic } \\
\text { Arch Graft }\end{array}$ & 4 & $\mathrm{~N} / \mathrm{A}$ & \\
\hline 10 & 39 & M & $\begin{array}{l}\text { Type A dissection } \\
\text { s/p TEVAR }\end{array}$ & TAA Repair & Unknown & $\mathrm{N} / \mathrm{A}$ & \\
\hline II & 63 & $\mathrm{~F}$ & $\mathrm{Al} / \mathrm{AS}$ & Redo AVR/Arch Repair & 6 & $\mathrm{~N} / \mathrm{A}$ & \\
\hline 12 & 76 & M & $\begin{array}{l}\text { Bio-AVR and } \\
\text { native MV } \\
\text { endocarditis }\end{array}$ & $\begin{array}{l}\text { AVR;TV repair; Patch to RA/Ao } \\
\text { fistula; Patch to Aortic root and } \\
\text { LVOT; pacemaker lead/generator } \\
\text { extraction; gastric hernia repair }\end{array}$ & 25 & N/A & $\begin{array}{l}\text { Death in ICU on Comfort } \\
\text { Care }\end{array}$ \\
\hline 13 & 60 & $M$ & $\mathrm{TR}$ & $\begin{array}{l}\text { Redo Sternotomy;TV repair; } \\
\text { MAZE }\end{array}$ & 10 & No Growth & \\
\hline 14 & 57 & M & $M R$ & MV Repair & 7 & No Growth & \\
\hline 15 & 52 & $M$ & $\begin{array}{l}\text { Ascending Aortic } \\
\text { Aneurysm; Redo } \\
\text { AVR }\end{array}$ & $\begin{array}{l}\text { Ascending Aortic Aneurysm/ } \\
\text { Redo AVR/patch repair aortic } \\
\text { aneurysm/Complication of post- } \\
\text { op tamponade and hemothorax } \\
\text { evacuation }\end{array}$ & 7 & $\mathrm{~N} / \mathrm{A}$ & \\
\hline 16 & 62 & M & $\mathrm{TR}$ & TVR & 7 & N/A & \\
\hline 17 & 70 & $M$ & Al;TR;MR & CABG; MVR;AVR & 6 & $\mathrm{~N} / \mathrm{A}$ & $\begin{array}{l}\text { Death in ICU on Comfort } \\
\text { Care }\end{array}$ \\
\hline 18 & 28 & $M$ & $\begin{array}{l}\text { Rheumatic Heart } \\
\text { Disease s/p } \\
\text { mechanical AV } \\
\text { thrombosis with } \\
\text { MR }\end{array}$ & AVR; MV Repair & 10 & N/A & \\
\hline 19 & 62 & $M$ & $\begin{array}{l}\text { Stenosis of Bio- } \\
\text { AVR and pulm } \\
\text { HTN }\end{array}$ & Redo AVR & I & $\begin{array}{l}\text { Grew Staph } \\
\text { epidiermidis }\end{array}$ & \\
\hline
\end{tabular}

'F, female; M, male; AS, aortic stenosis; TAVR, transcatheter aortic valve replacement; ECMO, extracorporeal membrane oxygenation; MV, mitral valve; IABP, intra-aortic balloon pump; MVR, mitral valve replacement; CHF, congestive heart failure; LVAD, left ventricular assist device; MR, mitral regurgitation; TAA, thoracic aortic aneurysm; TR, tricuspid regurgitation; ASD, atrial septal defect; LAA, left atrial appendage; TEVAR, thoracic endovascular aortic repair; Al, aortic insufficiency; AVR, aortic valve replacement; Bio, bioprosthetic; LV, left ventricle; CVC, central venous catheter; ICU, intensive care unit; CABG, coronary artery bypass graft; Pulm, pulmonary; HTN, hypertension; N/A, not applicable

\section{Discussion}

2-octyl cyanoacrylate can be applied during central line placement. Adverse skin reactions associated with the 2-octyl cyanoacrylate are uncommon and were not observed in our study. The bacterial catheter colonization rate of $20 \%$ in our study was similar to the findings of Reny et al. ${ }^{16}$ where they observed a $25 \%$ bacterial catheter colonization rate among critically ill patients. ${ }^{16}$ Raman Spectroscopy and Electron Microscopy demonstrated no evidence of new product formation or microstructural degradation between 2-octyl cyanoacrylate and the central venous catheter. Furthermore, subsequent removal of the 2-octyl cyanoacrylate with acetone did not impact the material integrity of the central venous catheters. These findings support the suitability of using 2-octyl cyanoacrylate with antimicrobial coated polyurethane multilumen catheters (Figure $2 \& 3$ ). This study was built around our primary objective of determining if the use of 2-octyl cyanoacrylate was feasible in the use of sealing catheter/skin insertion sites. Additionally, our primary endpoint was built around determining the duration of time in days that the adhesive seal remained intact. This assessment was accomplished visually with manual inspection which was most accurate when the dressing was removed for a regular 
dressing change. This resulted in days where visual inspection was impossible and could not be accurately assessed. In these cases, the duration of maintenance of seal integrity was reported as the last day the seal was known to be intact. Complete failure of the adhesive was relatively easy to interpret, but minor cracks or subtle imperfections were considerably less so. It is unclear if such problems with the adhesive were occurring and to the extent they represented failure of the sterile barrier.

Table 2 Patient specific data for days before the adhesive cracked or the catheter was removed

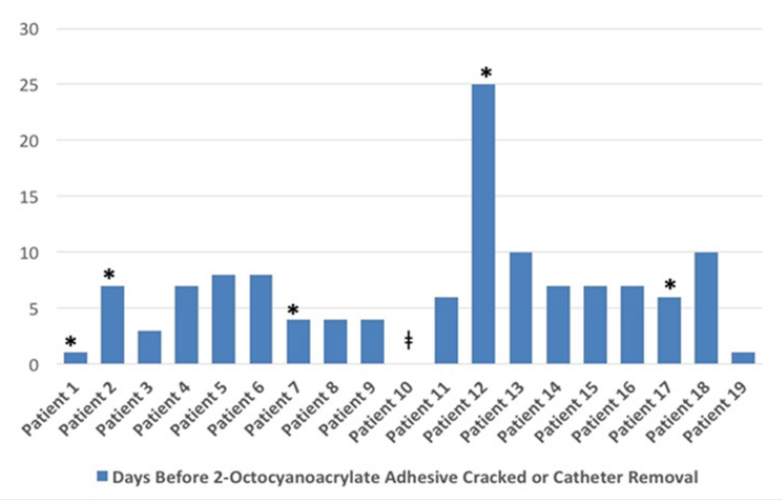

* Patient death unrelated to central venous catheter or tissue adhesive. $\neq$, Unidentified loss of seal date.

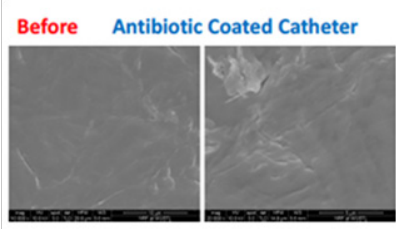

After

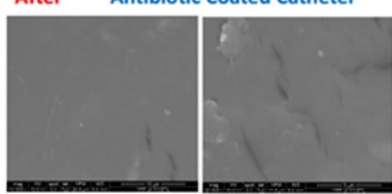

Figure 2 Electron microscopy demonstrated no chemical or structural changes to the catheter from the 2-octyl cyanoacrylate adhesive following its removal with acetone after a 10 -day dwell period.
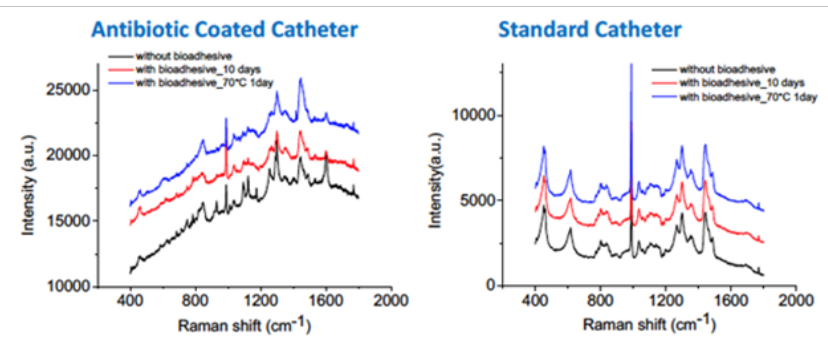

Figure 3 Raman spectroscopy demonstrated no chemical or structura changes to the catheter from the 2-octyl cyanoacrylate adhesive following its removal with acetone after a 10 -day dwell period.

\section{Conclusion}

The use of tissue adhesive to secure central venous catheters remains a promising solution to a pressing challenge. The success of this method of catheter sealing is logically related to the duration of time the seal remains intact but this has not been determined. The ability of the tissue adhesive-central venous catheter interface to remain sealed for an average of 7 days is an encouraging find that lends credence to the idea that such an intervention could be successful. Different application techniques or reapplication of 2-octyl cyanoacrylate could be important to enhance clinical utility. Since this study was small and the endpoints used do not allow us to make determinations regarding CLABSI or catheter colonization rates.

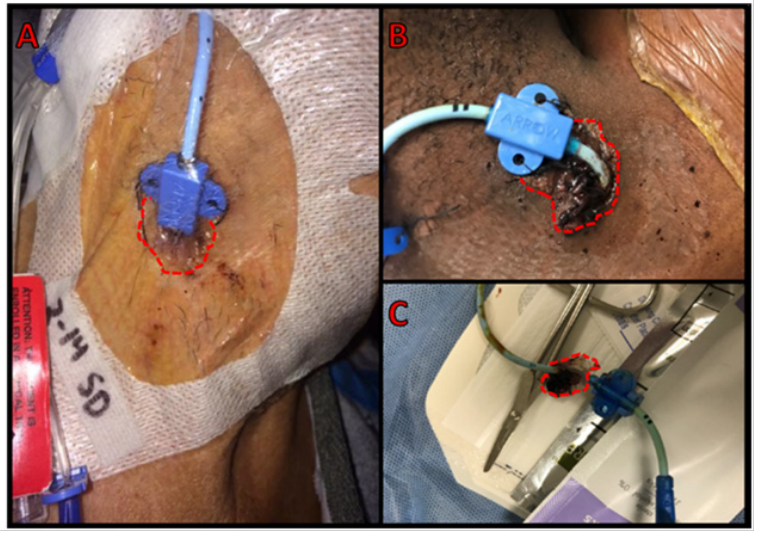

Figure 4 Multilumen central venous catheters (A) with the skin insertion point covered by 2-octyl cyanoacrylate adhesive (outlined in red dashed line in all pictures) and (B) skin insertion point covered by adhesive with semipermeable dressing removed $(C)$ and a catheter following removal with adhesive still attached.

The benefits of this intervention remain theoretical, although supported by a biologically plausible mechanism of action, and need large human trials to reveal any benefit. An ideal endpoint would examine the incidence of CLABSI but such a large, trial may be challenging to complete. Potential surrogate measures such as bacterial colonization of the catheter may prove more attainable. This study should build the foundation for further trials.

\section{Acknowledgments}

Aaron B. Dahl, MD, Zachary Cohen, MD, David K. Warren, MD, MPH, and Thomas J. Graetz, MD,

\section{Conflicts of interest}

The authors declare no conflicts of interest.

\section{Funding}

None.

\section{References}

1. Dudeck MA, Weiner LM, Allen-Bridson K, et al. National Healthcare Safety Network (NHSN) Report, Data Summary for 2012, Deviceassociated Module. Am J Infect Control. 2013;41(12):1148-1166.

2. Mermel LA. Prevention of intravascular catheter-related infections. Ann Intern Med. 2000;132(5):391-402.

3. Moretti EW, Ofstead CL, Kristy RM, et al. Impact of central venous catheter type and methods on catheter-related colonization and bacteraemia. $J$ Hosp Infect. 2005;61(2):139-145.

4. Mermel LA. What is the predominant source of intravascular catheter infections? Clin Infect Dis. 2011;52(2):211-212.

5. O'Grady NP, Alexander M, Burns LA, et al. Guidelines for the Prevention of Intravascular Catheter-Related Infections, 2011. Centers for Disease Control and Prevention. 2011;1-82. 
6. Simonova G, Rickard CM, Dunster KR, et al. Cyanoacrylate tissue adhesives - effective securement technique for intravascular catheters:in vitro testing of safety and feasibility. Anaesthesia intensive care. 2012;40(3):460-466.

7. Wilkinson JN, Sheikh N, Jayamaha J. Tissue adhesive as an alternative to sutures for securing central venous catheters. Anaesthesia. 2007;62(9):969-970.

8. Eaglstein WH, Sullivan T. Cyanoacrylates for skin closure. Dermatol clin. 2005;23(2):193-198.

9. Fraeman KH, Reynolds MW, Vaughn BB, et al. Patient outcomes associated with 2-octyl cyanoacrylate topical skin adhesive in coronary artery bypass graft surgery. Surg Infect (Larchmt). 2011;12(4):307-316.

10. Eymann R, Kiefer M. Glue instead of stitches:a minor change of the operative technique with a serious impact on the shunt infection rate. Acta Neurochir Suppl. 2010;106:87-89.

11. Wilkinson JN, Fitz-Henry J. Securing epidural catheters with Histoacryl glue. Anaesthesia. 2008;63(3):324.
12. Maki DG, Jarrett F, Sarafin HW. A Semiquantitative Culture Method for Identificaiton of Catheter-Related Infection in the Burn Patient. J Surg Res. 1977;22:8.

13. Singer AJ, Thode HC. A review of the literature on octylcyanoacrylate tissue adhesive. Am J Surg. 2004;187(2):238-248.

14. Hivnor CM, Hudkins ML. Allergic contact dermatitis after postsurgical repair with 2-octylcyanoacrylate. Arch Dermatol. 2008;144(6):814 815.

15. Dragu A, Unglaub F, Schwarz S, et al. Foreign body reaction after usage of tissue adhesives for skin closure:a case report and review of the literature. Arch Orthop Trauma Surg. 2009;129(2):167-169.

16. Reny J, Vuagnat A, Ract C, et al. Diagnosis and follow-up of infections in intensive care patients: Value of C-reactive protein caompared with other clinical and biological variables. Crit Care Med. 2002;30(3):529535 